\title{
Abstracts of Papers Presented at the Fifteenth Conference of European Endocrinologists
}

\author{
September 9-14, 1990, Leuven, Belgium
}

Edited by lan W. Henderson and Carolyn F. Deacon

\author{
Steroid Hormones: Actions and Interplay \\ Ecdysteroids \\ $1-14$ \\ Reproductive Endocrinology \\ 15-31 \\ Endocrine Control of Hydromineral Homeostasis \\ 32-89 \\ $90-116$ \\ Hormones and Metabolism \\ $117-148$
}

Abstract No.

Neuropeptides: Structure, Localization and Coexistence

(a) Vertebrates

149-197

(b) Invertebrates

$198-229$

230-250

Neuropeptides: Physiological Actions

$251-312$

Regulation of Hormone Synthesis and Release

$313-321$

Endocrine Responses to Stress

$322-349$

Applied and Methodological Aspects of Comparative Endocrinology

1. The Steroid Hormone of Sunlight Soltriol (Vitamin D): Receptors in Vertebrate Phylogeny and Role in Seasonal Regulation of Life. W. E. STUmPF AND H. J. Bidmon, Department of Cell Biology and Anatomy, University of North Carolina, Chapel Hill, North Carolina 27599.

In the past, vitamin D has been regarded as "the calcium homeostatic steroid hormone." However, autoradiographic studies with tritiated dihydroxycholecalciferol in rodents have demonstrated specific nuclear binding (receptors) in endocrine, neural, cardiovascular, glandular, reproductive, and immunological tissues, as well as in skin (Histochemistry 89, 209). Vitamin D-soltriol has been shown to affect pituitary TSH cells and pancreatic $\beta$ cells to elevate TSH and insulin blood levels. Extensive distribution of soltriol target cells has now been demonstrated in representatives of all vertebrate groups in the central nervous system and pituitary and in both female and male reproductive organs. Vitamin D-soltriol cannot be understood in the narrow sense of systemic and cellular calcium regulation alone; it is a steroid hormone of sunlight that influences seasonal adaptation of all vital functions that include development, growth and motor activities, sexual maturation and reproduction, immune responses, and cardiovascular and endocrine-autonomic regulation.

2. Comparative Studies of Corticosteroid Receptor Binding in Lower Vertebrates. A. GERHARD AND W. HANke, Zoological Institute II, Kaiserstr. 12, D-7500 Karlsruhe, Federal Republic of Germany.

The binding of different natural and synthetic corticosteroids to glucocorticoid receptors was investigated in several species of lower vertebrates, Myxine glutinosa (Cyclostomata), Cyprinus carpio, Oreochromis mossambicus (Teleostei), Ambystoma mexicanum, and Xenopus laevis (Amphibia). The dissociation constants ( $K_{D}$ ) and the maximal concentration of binding sites $\left(N_{\max }\right)$ were determined by Scatchard plot. The aim of the study was to answer the following questions: 1 . Is there an evolution of receptor specificity? 2 . Is there any obvious difference between a "mineralocorticoid" and a "glucocorticoid" receptor? The naturally occurring corticosteroids, aldosterone (A), corticosterone $(\mathrm{B})$, cortisol $(\mathrm{F})$ and 11-deoxycortisol (S) were bound by gill as well as by liver of the Atlantic hagfish, while the synthetic compounds dexamethasone (d) and triamcinolone acetonide (TA) were not. No differences in $K_{D}$ and $N_{\max }$ between gill and liver were observed. The binding hierarchy was: $A>B \geqslant F \geqslant S$. The binding affinities of the hormones in the two teleost in both organs were identical TA $>$ $\mathrm{D}>\mathrm{F}$. In carp organs binding of $\mathrm{F}$ was similar to that of $\mathrm{S}$. Comparing both species, carp in general showed a higher $N_{\max }$ than tilapia. In the liver of amphibians the order of binding was TA $>\mathrm{D}>\mathrm{B}>\mathrm{F}$. Binding of A was found in Axolotl liver and binding of $S$ in Xenopus liver. In conclusion, the receptor does not seem to discriminate between the four natural corticosteroids in hagfish. The teleost and amphibian receptor showed an affinity for the 
encoding the cleavage region (gly-lys-arg) between GnRH and the putative salmon GnRH associated peptide (GAP) is identical with the rat sequence. The DNA sequence upstream from GnRH encodes a 23amino-acid-long hydrophobic signal peptide sequence the same as the rat gene. Structural properties of the GnRH gene have thus been well conserved.

82. Steroids in Seminal Fluid in the Rainbow Trout. M. LoIr, C. Labbe, AND D. Vizziano, Physiologie des Poissons, INRA, 35042 Rennes, France.

$17 \alpha, 20 \beta$-dihydroprogesterone $(17,20 \mathrm{P})$, testosterone $(\mathrm{T}), 11$ ketotestosterone $(\mathrm{KT})$ in the conjugated $(\mathrm{C})$ and unconjugated (U) forms and 17 $\alpha$-hydroxyprogesterone (17P4) were assayed by RIA in blood plasma (BP) and in seminal fluid (SF) collected from 46 males at the beginning of the spawning season. Plasma levels $(\mathrm{ng} / \mathrm{ml})$ were 17P4: 1.86; U-17,20P: 8.42, C-17,20P: 0.67; U-T: 86.9, C-T: 14.45; U-KT: 88.3, C-KT: 9.0. Levels in SF were 17P4: undetectable; U-17,20P: 2.17, C-17,20P: 0.28; U-T: 10.18, C-T: 103.83; U-KT: 10.15, C-KT: 12.03. While the levels in BP and in SF of the U form for each of the two androgens were correlated, they were not for 17,20P. For each of the three steroids, the levels in BP and in SF of the $\mathrm{C}$ form were not correlated. Incubations of washed spermatozoa in the presence of 17P4 and P4 confirmed that sperm cells possess a 203HSD activity unaffected by s-GtH2, but no $17 \alpha$ OHase activity. Whatever the amount of $17,20 \mathrm{P}$ produced by the spermatozoa, this steroid accumulated only in the extracellular medium but not in the sperm cells. Incubations of fractions of spermatozoa prepared after nitrogen cavitation indicated that the 203HSD activity was localized in the sperm membrane. After 17,20P levels in semen collected from the testis to the posterior part of vas deferens, the apparent production of $17,20 \mathrm{P}$ by $10^{8}$ spermatozoa decreased progressively along the genital tract, reflecting either a decrease of $17 \mathrm{P} 4$ provided by the testis or a reabsorption of $17,20 \mathrm{P}$ from the SF to the BP. Present and previous results suggest that $17,20 \mathrm{P}$ in SF comes mainly from spermatozoa, while most plasma 17,20P originates from the interstitial cells.

83. Sex Steroids in Male Rainbow Trout. R. Schulz, ${ }^{*}$ M. Andriske, $†$ P. Lembee,$\dagger$ And V. Blum, $\dagger$ Research Groups for Comparative Endocrinology, *Padualaan 8, NL-3584 CH Utrecht, the Netherlands, and †Universitätsstr. 150, D-4630 Bochum 1, West Germany.

Plasma sex steroid and maturational gonadotropic hormone (GTH) levels peak during the spawning season of male rainbow trout. This is difficult to understand in the light of the negative feedback sex steroids exert on the pituitary. Since changes in gonadal GTH sensitivity may be related to this phenomenon, testicular steroid output in vitro, stimulated by a pituitary extract (PE) from mature Oncorhynchus spec., was studied at different stages of the reproductive cycle. The sensitivity to PE (maximal stimulated/basal steroid secretion; PE doseevoking half-maximal response $\left[\mathrm{ED}_{50}\right]$ ) was highest at the end of spermatogenesis and at maturity, but was low in immature or spent fish. $\mathrm{ED}_{50}$ values for $17 \alpha$-hydroxy, $20 \beta$-dihydroprogesterone were at least three times higher than for the three androgens (11-ketotestosterone, testosterone, 11ß-hydroxytestosterone) quantified. A GTH treatment that augmented plasma steroid levels of tissue donors further increased testicular steroid output in response to $\mathrm{PE}$ in vitro in fish at the end of spermatogenesis and in the spawning season, indicating that, in contrast to mammals, there is no GTH-mediated down-regulation of testicular steroid production in postpuberal fish. Attenuation of GTH bioactivity in fully mature fish by passive immunization with a GTH antiserum raised against SGA-GTH (Syndel, Canada) decreased plasma steroid levels and sensitized testicular steroid secretory responses to $\mathrm{PE}$, which was reflected in the secretion of more steroid and in lower $\mathrm{ED}_{50}$ values. It therefore appears that, in addition to being a target site within the brain-pituitary-gonad axis, the testicular steroidogenic system is an active part of this regulatory system, adapting in maturing and mature fish under decreased as well as increased GTH stimulation toward the increased production of steroid. This may give insight into how steroid and GTH plasma levels interact.

84. Effect of D-Ala $a^{6}$ Pro ${ }^{9}-N$-ethylamide-LHRH (LHRH-A) and Pimozide on the Sterlet (Acipenser ruthenus L.) Ovary Steroid-Producing Cells. I. A. Barannikova, E. V. Vasiljeva, And V. P. Duubin, Physiological Institute, Leningrad University, Leningrad, USSR.

In prespawning female sterlet administration of pimozide (I, PIM, 5-2,5 mg/kg BW), a dopamine receptor antagonist, LHRH-A (II, $5 \mathrm{mkg} / \mathrm{kg} \mathrm{BW}$ ), and PIM + LHRH-A (III, same doses) lead to ovulation of some fish

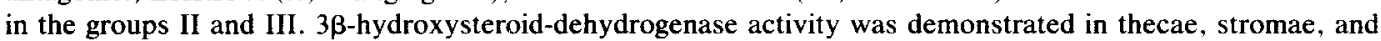
granulosa cells of the pre- and postovulatory follicles from fish of all three groups $45-50 \mathrm{hr}$ after injections. Ultrastructure of steroid cells of sterlet (Chondrostei) is typical with the cytoplasm containing mitochondria (m) with tubulo-vesicular cristae, smooth, and rough endoplasmic reticulum (er), and lipid droplets. After the 